\title{
P02-1-51 Poster session
}

\section{Role of Lrrn3, a brain-enriched transmembrane protein in neural development}

\author{
Maiko Nakamura, Minoru Hatayama, Hayato Matsunaga, Shinsuke Nakagawa, Jun Aruga
}

Department of Medical Pharmacology, Nagasaki University Institute of Biomedical Sciences, Japan

Accumulating evidences indicate that Leucine-rich repeat containing transmembrane proteins play critical roles in brain development and its higher functions. They mediate various cellular processes such as neurite development, synapse formation, maintenance of synaptic functions as adhesion molecules, and transmembrane signaling as receptors. We noticed that some Leucine-rich repeat containing transmembrane proteins are potentially expressed not only in neurons but also in brain vasculatures through bioinformatic analysis. One of the candidate protein was Leucine-rich repeat containing neuronal 3 (Lrrn3, also known as Nlrr3). In a previous study, human LRRN3 is associated with the cortical gray matter thickness in transcriptomics (Kochunov et al., 2013). However, little is known about its physiological role in brain. To address this point, we analyzed the CNS phenotype of Lrrn3-deficient mice. In our initial characterization, a part of Lrrn3-deficient mice showed severe developmental delay and behavioral abnormalities. Morphological analysis revealed structural alterations in Lrrn3-deficient brain. Together with in vitro and in vivo overexpression analyses, we examined the role of Lrrn3 in neuronal development. 